\title{
Adaptive neuro fuzzy determination of impactful factors on non-uniformity of polished surface
}

\author{
Dalibor Petkovic \\ University of Niš, Pedagogical Faculty in Vranje, Partizanska 14, 17500 Vranje, Serbia \\ Email: dalibortc@gmail.com
}

\begin{abstract}
How to cite this paper: Dalibor Petkovic (2019) Adaptive neuro fuzzy determination of impactful factors on non-uniformity of polished surface. Journal of the Institute of Electronics and Computer, 1, 9-16. https://doi.org/10.33969/JIEC.2019.11002

Received: September 10, 2019

Accepted: October 20, 2019

Published: November 7, 2019

Copyright (C) 2019 by author(s) and Institute of Electronics and Computer. This work is licensed under the Creative Commons Attribution International License (CC BY 4.0).

http://creativecommons.org/licenses/by/4.0/

(c) (i) Open Access
\end{abstract}

\begin{abstract}
In this study, the neuro fuzzy logic selection of the most influential parameters for non-uniformity of polished surface was performed. The influence of three parameters was analyzed: carrier load, thickness and the pads elastic modulus. Adaptive neuro fuzzy logic or ANFIS was used for establishing the nonlinear relationships between the three parameters and the non-uniformity polished surface. Dataset for training and testing procedure was extracted by finite element model. According to the regression models, thickness of pad has the greatest influence on the non-uniformity. Moreover, elastic modulus and pad thickness are the optimal combination of non-uniformity. Results could be useful in the improvement of chemical polishing process.
\end{abstract}

Keywords

Wafer, Chemical, Polishing Process, Non-uniformity, Neuro Fuzzy

\section{Introduction}

Chemical polishing process is the process which is based on applied pressure on surface top of a wafer carrier. This pressure is used for the pad polishing and also for the slurry injection in order to polish the metallic film. Development of semiconductor industry leads to the reduction of wafer size and circuit patterns become more complex. Polishing process by chemical substances is the most important method for the non-uniformity. The first step in improvement of the non-uniformity is to analyze which parameters are most impactful on the non-uniformity.

It is rather difficult to achieve uniform surface texture and high convergence rates of surface roughness (CRSR) at the same time in the conventional optical polishing process. Under the dual-axis mode, the variation of co-rotating speed has less influence on the polishing quality, very high CRSR and uniform texture can be achieved under different co-rotating speeds [1]. The preparation of 
semiconductor silicon polished wafer is a multi-stage manufacturing process. The experimental results in article [2] showed that different pre-polishing processes have significant effects on SFQR (Site flatness front least square range) values of polished wafers. Theoretical analyses demonstrated that, in the process of megasonic-assisted chemical-mechanical polishing, the vibrations enable a part of idle particles, stored in the cells of the polishing pad, to squeeze into the contact areas [3]. The results in article [4] showed that megasonic assisted chemical mechanical polishing is a feasible method to improve polishing efficiency and surface quality. The need for polishing silicon wafer is to obtain a smooth surface for sprucing the semiconductor devices on the wafer surface [5]. Rapid progress in recent IC fabrication industry has increased the demand of tight specification of non-uniformity (NU) and surface polishing in silicon wafer planarization. Chemical-mechanical polishing (CMP) is currently the most popular method for IC wafer planarization. The NU has been estimated for evaluation of mechano-chemical polishing (MCP) parameters [6]. This study [7] investigated the synergistic effect of mechanical and chemical factors on the CMP performance of through-silicon via (TSV), and analyzed the experimental results from different aspects with a high-resolution surface-profile-measuring instrument, combined with Scanning Electron Microscopy (SEM) and other measurements, it was used to demonstrate the mechanism of non-uniformity and via dishing, and the experimental results indicate that via dishing and non-uniformity of wafers are strongly mitigated by combining high down force with low down force. This study [8] established a correlation mechanism between the Nano material removal and global uniformity of wafer surfaces, which provides theoretical and experimental framework for optimizing the slurry and the process parameters.

In this study, the neuro fuzzy logic [9] selection of the most influential parameters for non-uniformity of polished surface is performed. The influence of three parameters are analyzed: carrier load, thickness and the pads elastic modulus. Finite element model (FEM) is used for data extraction.

\section{Methodology}

\subsection{Chemical polishing process}

The basic purpose of chemical polishing process is the removal of material on water surface. Material removal rate (MRR) during the process could be expressed by Preston's equation:

$$
M R R=C_{p} P V
$$

where $\mathrm{P}$ is normal pressure, $\mathrm{V}$ is the relative velocity and $\mathrm{Cp}$ is Preston's constant. In above equation, the normal pressure can be controlled and $\mathrm{V}$ represent a vector velocity of a point on the wafer. Figure 1 shows the model of the chemical polishing process in this paper, where it is represented as a two-dimensional quasi-static model. 


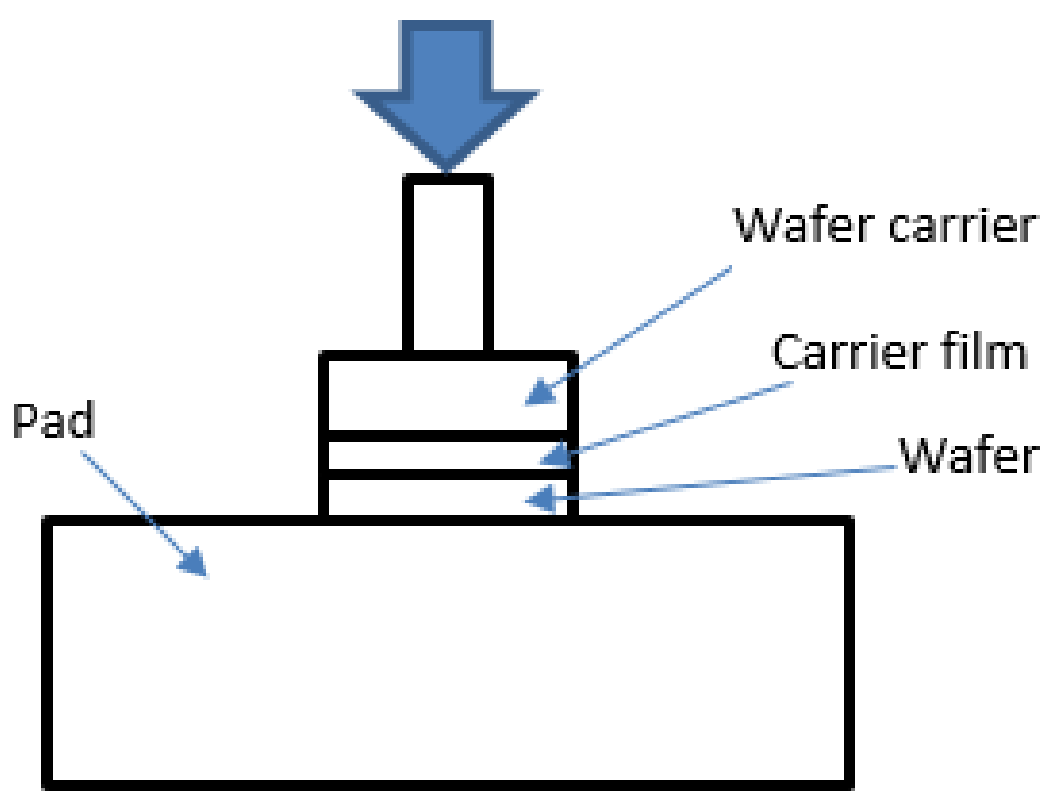

Figure 1. Illustration of chemical polishing process

According to the principle of minimum potential energy, finite model for a two-dimensional quasi-static wafer surface can be presented as follows:

$[K]\{\delta\}=\{Q\}(2)$

where $[\mathrm{K}]$ represents the elastic stiffness matrix, $\{\delta\}$ is the modal displacement vector and $\{Q\}$ is the nodal force vector.

\subsection{Data description}

Table 1 shows input and output parameters which are used in this study, based on finite element model defined in previous section. The analyzing is based on investigation in this paper [10]. As can be seen in Table 1, there are three inputs and one output. These input are carrier load, elastic modulus of pad and thickness of pad. The input parameters are varied in defined ranges according to the prescribed scheme. The non-uniformity can be expressed as:

$R=\frac{\sigma_{\max }}{\sigma_{c}}$

where $\sigma_{\max }$ is maximal von Mises stress on the wafer surface and $\sigma_{c}$ is the von Mises stress on the wafer center. 
Table 1. Input and output parameters [10]

\begin{tabular}{|c|c|c|c|}
\hline input 1 & input 2 & input 3 & output \\
\hline $\begin{array}{c}\text { Carrier load } \\
(\mathrm{MPa})\end{array}$ & $\begin{array}{c}\text { Elastic modulus of } \\
\text { pad }(\mathrm{MPa})\end{array}$ & $\begin{array}{l}\text { Thickness of } \\
\text { pad (mm) }\end{array}$ & $\begin{array}{l}\text { Non-uniformity } \\
\text { of wafer surface }\end{array}$ \\
\hline 0.03448 & 1.148 & 0.6985 & 1.793818 \\
\hline 0.03448 & 1.148 & 1.397 & 1.947089 \\
\hline 0.03448 & 1.148 & 2.0955 & 2.076029 \\
\hline 0.03448 & 2.2897 & 0.6985 & 1.716075 \\
\hline 0.03448 & 2.2897 & 1.397 & 1.866292 \\
\hline 0.03448 & 2.2897 & 2.0955 & 1.992638 \\
\hline 0.03448 & 3.4345 & 0.6985 & 1.676571 \\
\hline 0.03448 & 3.4345 & 1.397 & 1.822172 \\
\hline 0.03448 & 3.4345 & 2.0955 & 1.945409 \\
\hline 0.069 & 1.148 & 0.6985 & 1.866979 \\
\hline 0.069 & 1.148 & 1.397 & 2.016311 \\
\hline 0.069 & 1.148 & 2.0955 & 2.140857 \\
\hline 0.069 & 2.2897 & 0.6985 & 1.763887 \\
\hline 0.069 & 2.2897 & 1.397 & 1.923106 \\
\hline 0.069 & 2.2897 & 2.0955 & 2.053362 \\
\hline 0.069 & 3.4345 & 0.6985 & 1.711978 \\
\hline 0.069 & 3.4345 & 1.397 & 1.868825 \\
\hline 0.069 & 3.4345 & 2.0955 & 1.999974 \\
\hline 0.10345 & 1.148 & 0.6985 & 1.922428 \\
\hline 0.10345 & 1.148 & 1.397 & 2.05949 \\
\hline 0.10345 & 1.148 & 2.0955 & 2.177593 \\
\hline 0.10345 & 2.2897 & 0.6985 & 1.804646 \\
\hline 0.10345 & 2.2897 & 1.397 & 1.964958 \\
\hline 0.10345 & 2.2897 & 2.0955 & 2.095692 \\
\hline 0.10345 & 3.4345 & 0.6985 & 1.743665 \\
\hline 0.10345 & 3.4345 & 1.397 & 1.906079 \\
\hline 0.10345 & 3.4345 & 2.0955 & 2.039954 \\
\hline
\end{tabular}

\subsection{ANFIS methodology}

In this study, bell-shaped membership functions were chosen. Fuzzy logic toolbox in MATLAB was used for the entire process of training and evaluation of fuzzy inference system. Figure 2 shows an ANFIS structure with two inputs, but the same procedure should be performed for any other number of inputs. 


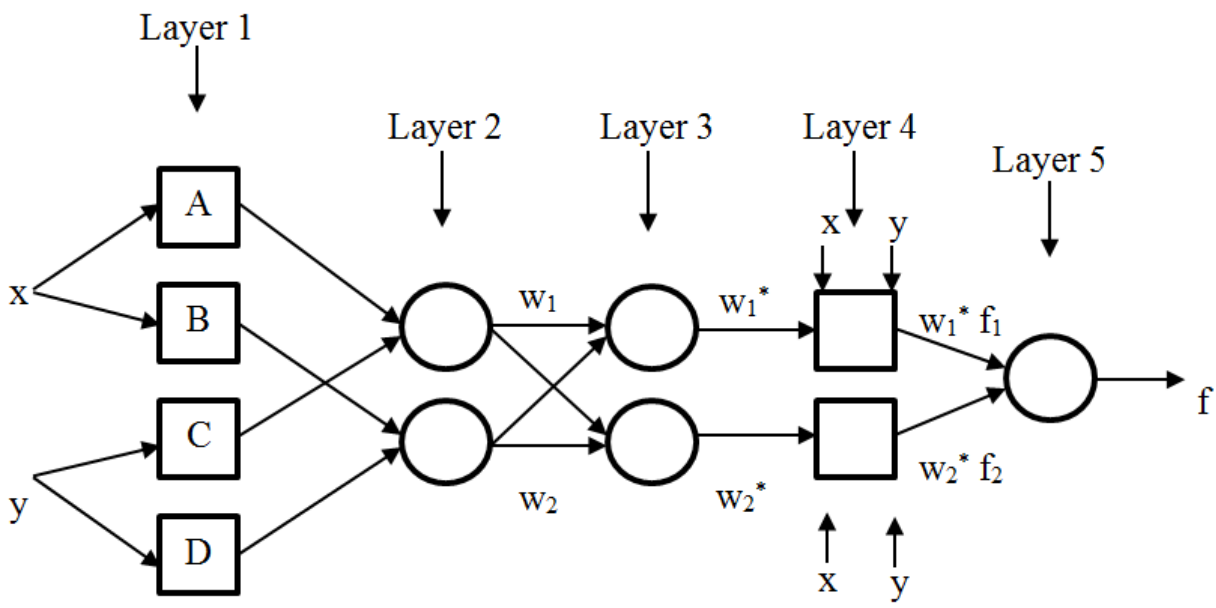

Figure 2. ANFIS structure

In this work, the first-order Sugeno model with two inputs and fuzzy IF-THEN rules of Takagi and Sugeno's type is used:

if $x$ is $A$ and $y$ is $C$ and $z$ is $E$ then $f_{1}=p_{1} x+q_{1} y+r_{1} z+t$

\section{Results}

Regression models for the non-uniformity prediction of wafer surface are modeled based on neuro fuzzy logic. In the beginning, three regression models are created as lsited bellow:

- Carrier load -->

RMSE (training) $=0.1328$, RMSE (testing) $=0.1250$

- Elastic modulus of pad -->

RMSE (training) $=0.1253$, RMSE (testing) $=0.1175$

- Thickness of pad -->

RMSE (training) $=0.0741$, RMSE (testing) $=0.0691$

These models are based on the separate single inputs. There are two root mean square (RMSE) values, one for training and one for testing of the ANFIS model. Training RMSE is used for selection of the most influential parameters on the non-uniformity prediction of the wafer surface. Smaller RMSE in training means higher influence of the parameter on the given output. Therefore, thickness of pad is the most influential parameter on the non-uniformity prediction of the wafer surface. Furthermore, the parameters could be combined in order to estimate the optimal combination for the non-uniformity. The obtained results for the two parameters' combinations are listed below:

- Carrier load and Elastic modulus of pad -->

RMSE (training) $=0.1180$, RMSE (testing) $=0.1110$

- Carrier load and Thickness of pad -->

RMSE (training) $=0.0614$, RMSE (testing) $=0.0577$

- Elastic modulus of pad and Thickness of pad -->

RMSE (training) $=0.0424$, RMSE (testing) $=0.0388$

One can see that the combination of elastic modulus and pad thickness is the most influental combination for the non-uniformity. Figures 3 and 4 presented the 
ANFIS predicted relationship between selected parameters and non-uniformioty of the wafer surface. One can note that there are strong nonlinearity between input and output parameters.

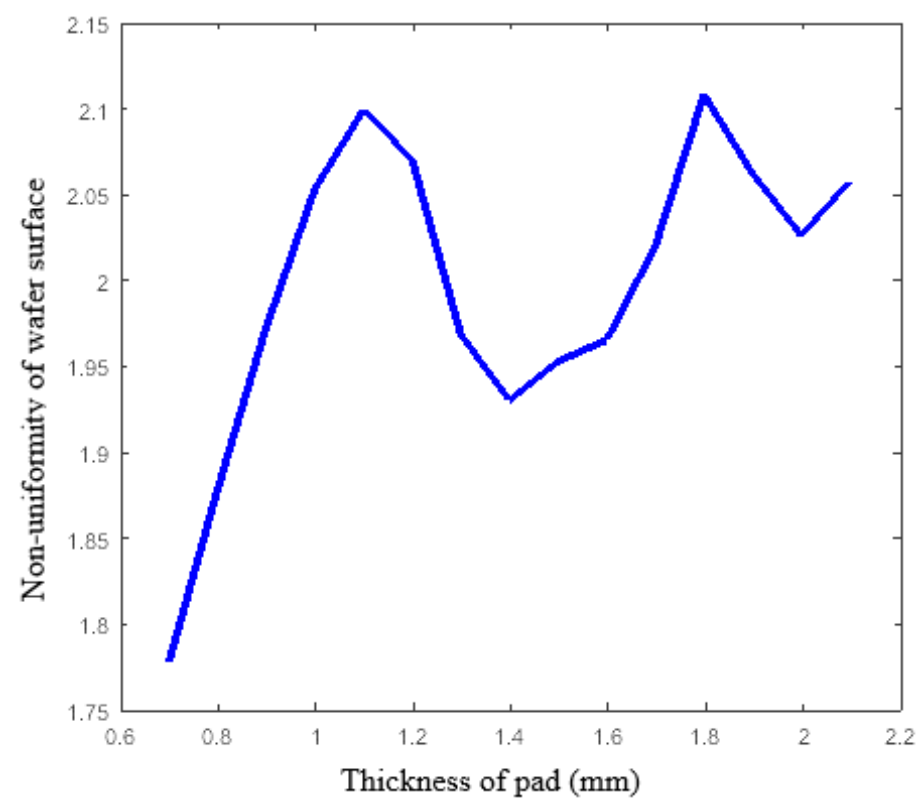

Figure 3. ANFIS predicted relationship between thickness of pad and non-uniformity

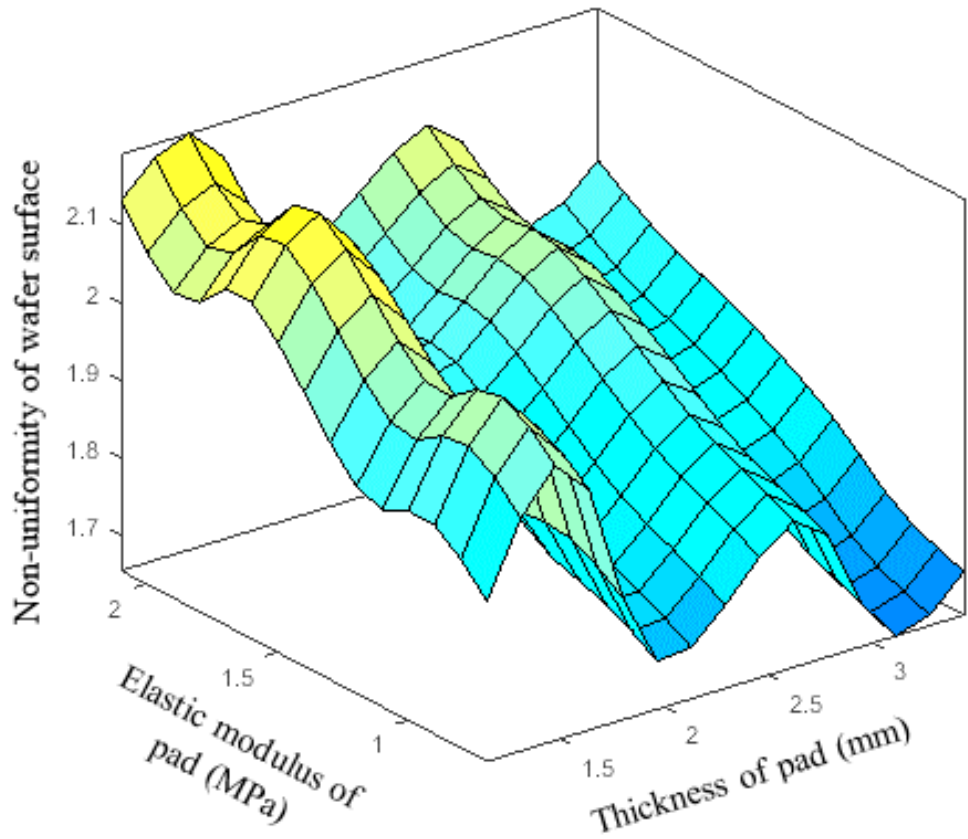

Figure 4. ANFIS predicted relationship among pad thickness, elastic modulus and non-uniformity 
Figures 5 and 6 show scatter plots of the ANFIS prediction of non-uniformoty of wafer surface for selected input combinations. Figure 5 shows prediction of non-uniformoty of wafer surface based on thickness of pad as input. There is medium correlation between FEM data and ANFIS predicted data according to the coefficient of determination $\mathrm{R}^{2}$. Figure 6 shows prediction of non-uniformoty of wafer surface based on thickness of pad and elastic modulus of pad as inputs. There is strong correlation between FEM data and ANFIS predicted data according to the coefficient of determination $\mathrm{R}^{2}$. Therefore it is sutiable if one use only selected input for prediction of the non-uniformity of the wafer surface.

Input 2

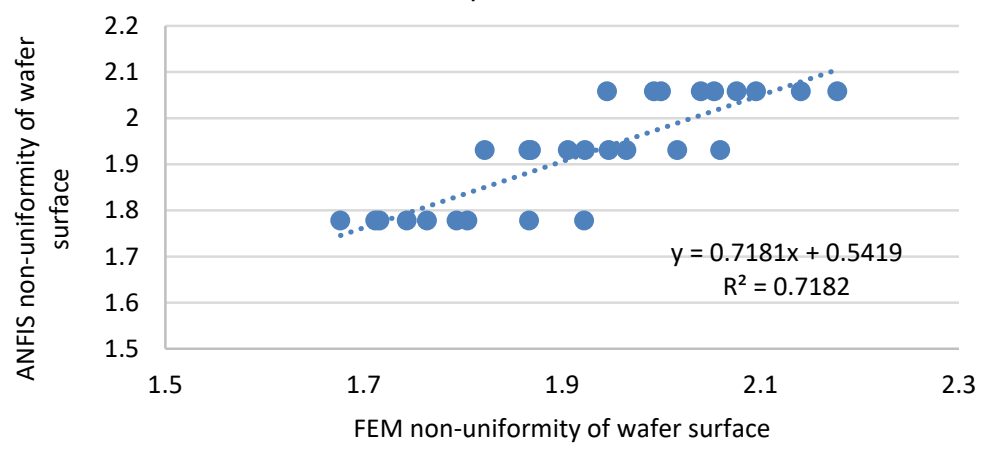

Figure 5. Scatter plot of ANFIS prediction for pad thickness as input

Input 2 and input 3

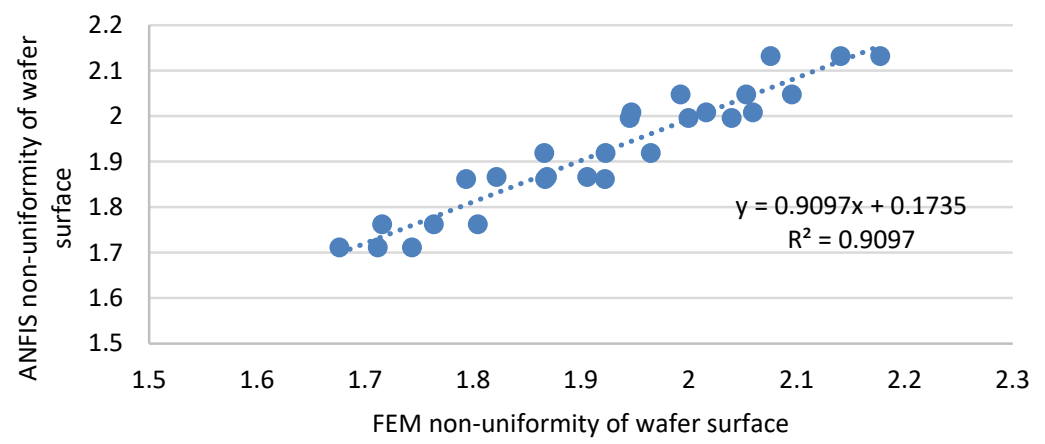

Figure 6. Scatter plot of ANFIS prediction for pad thickness and elastic modulus as inputs

\section{Conclusion}

This study presented a selection procedure of the most influential parameters for non-uniformity of polished 4ent model which was developed for data samples extraction for the ANFIS modeling. The influence of three parameters on the non-uniformity was analyzed: carrier load, thickness and the pads elastic modulus. 
According to the obtained results, thickness of pad has the greatest influence on the non-uniformity. Also combination of elastic modulus of pad and thickness of pad is the most optimal combination on the non-uniformity. The obtained results could be useful in the improvement of chemical polishing process.

\section{Conflicts of Interest}

There is no conflict of interest.

\section{References}

[1] Lu, A., Jin, T., Liu, Q., Guo, Z., Qu, M., Luo, H., \& Han, M. (2019). Modeling and prediction of surface topography and surface roughness in dual-axis wheel polishing of optical glass. International Journal of Machine Tools and Manufacture, 137, 13-29

[2] Zhong, G., Ning, Y., Zhou, Q., Bian, Y., Wang, X., Qu, X., ... \& Zhao, E. (2017). Influence of pre-polishing process on site flatness values of polished wafers. Materials Science in Semiconductor Processing, 68, 15-20.

[3] Li, L., He, Q., Zheng, M., Ren, Y., \& Li, X. (2019). Improvement in polishing effect of silicon wafer due to low-amplitude megasonic vibration assisting chemical-mechanical polishing. Journal of Materials Processing Technology, 263, 330-335

[4] Zhai, K., He, Q., Li, L., \& Ren, Y. (2017). Study on chemical mechanical polishing of silicon wafer with megasonic vibration assisted. Ultrasonics, 80 , 9-14.

[5] Pandey, K., \& Pandey, P. M. (2017). Chemically assisted polishing of monocrystalline silicon wafer $\mathrm{Si}(100)$ by DDMAF. Procedia engineering, 184, 178-184.

[6] Chen, C. C. A., Shu, L. S., \& Lee, S. R. (2003). Mechano-chemical polishing of silicon wafers. Journal of Materials Processing Technology, 140(1-3), 373-378.

[7] Rao, C., Wang, T., Wang, J., Liu, Y., \& Lu, X. (2016). Improvement of via dishing and non-uniformity in TSV chemical mechanical planarization. Microelectronic Engineering, 151, 38-46.

[8] Li, J., Wei, Z., Wang, T., Cheng, J., \& He, Q. (2017). A theoretical model incorporating both the nano-scale material removal and wafer global uniformity during planarization process. Thin Solid Films, 636, 240-246

[9] Jang, J.-S.R, ANFIS: Adaptive-Network-based Fuzzy Inference Systems, IEEE Trans. On Systems, Man, and Cybernetics (1993), Vol.23, 665-685.

[10] Lo, S. P., \& Lin, Y. Y. (2005). The prediction of wafer surface non-uniformity using FEM and ANFIS in the chemical mechanical polishing process. Journal of Materials Processing Technology, 168(2), 250-257. 